\title{
Professor Dr. Dr. Hans Schadewaldt in memoriam
}

\author{
Am 21. August dieses Jahres ist Prof. Dr. Dr. Hans Schadewaldt, Ordi- \\ narius in Düsseldorf, verstorben. Er war ohne Zweifel der weltweit \\ bedeutendste allergologische Medizinhistoriker.
}

D rofessor Schadewaldt hat die Allergologie lebenslang begleitet. Wer seine Habilitationsschrift über die „Geschichte der Allergie“ aus dem Jahr 1961 nicht kennt, dem fehlt eine der wesentlichen Grundlagen unseres Faches: Sie wurde von 1979 bis 1983 im DustriVerlag publiziert und umfasst vier Bände mit insgesamt 1817 Seiten und $4251 \mathrm{Zi}$ taten. Schadewaldt hat außerdem 1984, ebenfalls im Dustri-Verlag, die Geschichte der Deutschen Gesellschaft für Allergie- und Immunitätsforschung, heute DGAKI, der Jahre 1951 bis 1984 veröffentlicht. Schadewaldt war Hauptreferent auf zahlreichen allergologischen Tagungen und zeitlebens freundschaftlich mit seinen allergologischen Zeitgenossen, aber auch mit den jüngeren Allergologen verbunden. Es war nur selbstverständlich, dass ihm 1990, gemeinsam mit Prof. Dr. Erich Fuchs, die höchste Auszeichnung der DGAKI, die KarlHansen-Medaille, verliehen wurde.

\section{Von Cottbus über Umwege nach Düsseldorf}

Hans Schadewaldt wurde am 7. Mai 1923 in Cottbus geboren. Wahrscheinlich ist es nicht unwichtig für das Verständnis seiner späteren beruflichen Orientierung, dass sein Großvater Arzt, sein Vater Journalist und Chefredakteur der lokalen Zeitung und sein Onkel der renommierte Altphilologe Wolfgang Schadewaldt waren. Schadewaldt war neben der Philologie lebenslang auch der Marine verbunden, beruhend wohl auf seinen Erfahrungen als Seekadett in den Jahren 1940 bis 1945 .

Schadewaldt legte sein medizinisches Staatsexamen 1949 an der Universität Freiburg im Breisgau ab. Bereits die Dissertation war einem medizinhistorischen Thema gewidmet. Seine philologischen Interessen ließen ihn von da an lebens-

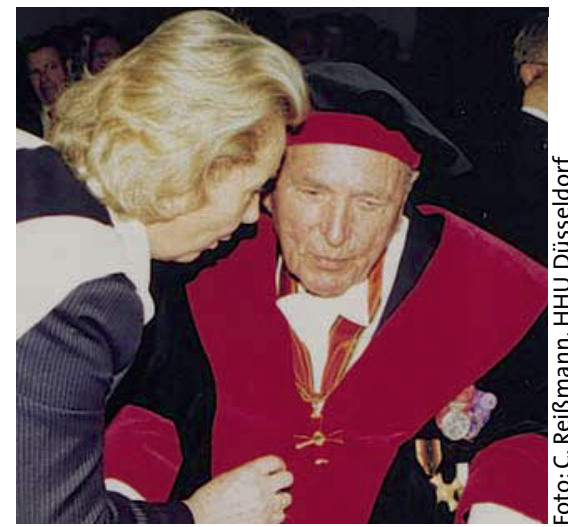

Prof. Dr. med. Dr. h.c. Hans Schadewaldt (*7. Mai 1923, † 21. August 2009) bei der Feier zu seinem 85. Geburtstag zusammen mit seiner Frau Rosemarie

lang nicht mehr los. Doch vorab absolvierte er eine klinische Weiterbildung von 1950 bis 1954 an der Kinderklinik der Universität Tübingen. Im Jahr 1955 übernahm Schadewaldt die Leitung der Wissenschaftlichen Literaturabteilung der CIBA AG in Wehr, Baden, und wurde damit auch Redakteur der international renommierten CIBA-Fachzeitschrift. Im Jahr 1961 habilitierte er sich mit der erwähnten Arbeit zur „Geschichte der Allergie" an der Universität Freiburg. Zwei Jahre später, 1963, wurde Schadewaldt als außerordentlicher Professor für Geschichte der Medizin an die Medizinische Akademie Düsseldorf berufen und 1965 anlässlich der Gründung der Universität Düsseldorf zum ordentlichen Professor ernannt. In den Jahren 1976 und 1977 war er Dekan der medizinischen Fakultät.

Von 1985 bis 1992 war Schadewaldt Präsident der Société Internationale d'Histoire de la Médecine und richtete 1986 den Internationalen Kongress für Medizingeschichte in Düsseldorf aus. Von 1990 bis 1993 war er Präsident der Nordrhein-Westfälischen
Akademie der Wissenschaften und leitete bis 1991 als Emeritus kommissarisch das Institut für Geschichte der Medizin. Hans Schadewaldt hat 770 wissenschaftliche Arbeiten veröffentlicht und über 290 Promotionen betreut.

\section{Akademischer „Hansdampf“ mit vielen Interessen}

Schadewaldt hatte ein sehr breites Interesse an medizinhistorischen Themen, wobei aber immer wieder die Detailgenauigkeit beeindruckte - Beleg für seinen unglaublichen Fleiß und sein großes Geschick in der Auffindung auch entlegener Quellen. In seinem enormen Oeuvre finden sich neben allergologischen Beiträgen auch zahllose Artikel zu etymologischen, gynäkologischen, infektiologischen, pädiatrischen, maritimen, pharmakologischen, homöopathischen und vielen anderen Themen, einschließlich der Würdigung bedeutender Wissenschaftler der Medizingeschichte. Er sei „ein akademischer Hansdampf“, neckten ihn seine Kollegen.

Schadewaldt widmete sich außerdem zahlreichen anderen Themen, wie der Betreuung der Grafiksammlung „Mensch und Tod“ der Heinrich-HeineUniversität, Düsseldorf. Diese Sammlung umfasst aktuell 3.000 Originalgrafiken und Zeichnungen zur Todesthematik und zählt damit zu den weltweit größten ihrer Art. Sie beinhaltet eine fast vollständige Reihe repräsentativer Totentanzzyklen, von der wohlbekannten Holzschnittfolge Hans Holbeins d. J. (1497/98-1543) bis zu hochaktuellen Totentänzen zeitgenössischer Künstler, sowie eine Fülle symbolischer und allegorischer Einzeldarstellungen zum Themenkomplex Vergänglichkeit, Sterben und Tod, vom Ende des 15. Jahrhunderts bis auf unsere Tage.

Mit Professor Schadewaldt hat Düsseldorf einen Wissenschaftler von Weltruf verloren, der sich auch für die Rettung des alten Landtags und für die bildende Kunst nachhaltig engagiert hat. Schadewaldt starb 86-jährig nach langer, schwerer Krankheit am 21. August 2009. Wir Allergologen dürfen nicht vergessen, dass uns der weltweit bedeutendste allergologische Medizinhistoriker verlassen hat.

Prof. Dr. Gerhard SchultzeWerninghaus, Bochum 